\section{Padrões alimentares e fatores associados entre crianças de um a seis anos de um município do Sul do Brasil}

\author{
Dietary patterns and associated factors among \\ children one to six years of age in a city in \\ southern Brazil
}

\author{
Patrón alimentario y factores asociados en niños \\ de uno a seis años en una ciudad \\ del sur de Brasil
}

Rosangela de Leon Veleda de Souza 1

Samanta Wink Madruga 2

Denise Petrucci Gigante ${ }^{1}$

Iná S. Santos 3

Aluísio J. D. Barros 3

Maria Cecília Formoso Assunção ${ }^{1}$

\footnotetext{
1 Programa de Pós-graduação em Nutrição e Alimentos, Universidade Federal de Pelotas, Pelotas, Brasil. 2 Programa de Pós-graduação em Educação Física. Universidade Federal de Pelotas, Pelotas, Brasil. 3 Programa de Pós-

graduação em Epidemiologia, Universidade Federal de Pelotas, Pelotas, Brasil.

Correspondência R. L. V. Souza Programa de Pós-graduação em Nutrição e Alimentos, Universidade Federal de Pelotas.

Rua Gomes Carneiro 1, Campus Anglo, Pelotas, RS 96010-610, Brasil.

rlveleda@yahoo.com.br
}

\begin{abstract}
This study aimed to describe dietary patterns and to investigate associations with demographic and socioeconomic factors among children one to six years of age in the city of Pelotas, Rio Grande do Sul State, Brazil. Pearson correlation was used to group different foods. Dietary patterns were constructed using principal components analysis (PCA). Associations were established with the Kruskal Wallis test $(\alpha=0.05)$. The study evaluated 667 children. Five dietary patterns were identified: "vegetables", "traditional" (bread, butter/margarine, rice/pasta, coffee, sugar), "sweets and sausages", "snacks" (dairy products, chocolate, cookies, and juice), and "fruits". Children of mothers with more schooling and higher income showed greater adherence to "vegetables" and "fruits". The "traditional" pattern was more common in children of mothers with less education and lower family income. The "vegetables" and "traditional" patterns showed greater variance. Families' socioeconomic status played a key role in determining children's dietary patterns.
\end{abstract}

Feeding Behavior; Child; Preschool Child; Statistical Factor Analysis

\section{Resumo}

O estudo teve como objetivos descrever padrões alimentares e investigar a associação com fatores demográficos e socioeconômicos entre crianças de um a seis anos na cidade de Pelotas, Rio Grande do Sul, Brasil. Foi utilizada a correlação de Pearson para agrupar os alimentos. Os padrões alimentares foram construídos por meio da análise de componentes principais. As associações foram verificadas usando-se o teste de KruskalWallis $(\alpha=0,05)$. Foram avaliadas 667 crianças. Identificou-se cinco padrões alimentares: "vegetais", "tradicional" (pão, margarinal margarina, arroz/massa, café, açúcar), "guloseimas e embutidos", "lanches" (laticinios, achocolatados, biscoitos e sucos) e "frutas". Os padrões "vegetais" e "frutas"tiveram maior adesão entre as crianças cujas mães tinham maior escolaridade e renda familiar. O padrão "tradicional" foi o mais observado entre crianças cujas mães tinham menor escolaridade e renda familiar. Os padrões "vegetais" e "tradicional" apresentaram o maior percentual de variância. As condições socioeconômicas das famílias exercem um papel fundamental na determinação do padrão alimentar das crianças.

Comportamento Alimentar; Criança; Pré-Escolares; Análise Fatorial 


\section{Introdução}

A alimentação nos primeiros anos de vida é de fundamental importância para uma nutrição adequada, tanto em aspectos qualitativos quanto quantitativos. Devido às suas características biológicas, os pré-escolares merecem atenção especial, já que uma alimentação inadequada pode colocar em risco seu crescimento e desenvolvimento ${ }^{1,2}$.

O perfil do padrão alimentar brasileiro vem sofrendo alterações desde a década de 90. A população tem adotado uma dieta com elevada proporção de gordura saturada, carboidratos simples e com baixo teor de carboidratos complexos, fibras, vitaminas e minerais, caracterizando um modelo ocidental de alimentação ${ }^{3}$. Esse padrão ainda evidencia dois cenários distintos em que a dieta conta com excessos nutricionais e inadequações alimentares 4 .

Há evidências de que os hábitos alimentares adquiridos durante a infância influenciam a saúde durante a adolescência e na vida adulta $5,6,7,8$. É nos primeiros anos de vida que o comportamento alimentar é estabelecido e tende a mostrar estabilidade em longo prazo ${ }^{9}$. A partir dessas constatações fica evidente a importância do conhecimento dos padrões alimentares para avaliar a necessidade de intervenções precoces durante a infância, no intuito de promover uma melhor qualidade de vida durante a adolescência e a fase adulta.

Padrões alimentares podem ser definidos como “...um conjunto ou grupo de alimentos consumidos por uma dada população" 10 (p. 312). Estudos para a identificação desses padrões utilizando análise de componentes principais (PCA) têm sido realizados principalmente em populações adultas 11. No Brasil, trabalhos que identifiquem padrões alimentares em crianças usando-se essa metodologia ainda são escassos 12,13,14,15. O estudo dos padrões alimentares, identificados por meio desse método, vem sendo amplamente utilizado como uma alternativa a estudos que levam em consideração a ingestão de alimentos ou nutrientes, isoladamente 16 .

Para o desenvolvimento de intervenções com a finalidade de melhorar a dieta dos pré-escolares é importante identificar, além dos seus padrões alimentares, os fatores que os determinam. O presente trabalho tem o objetivo de identificar, descrever e avaliar a associação entre os padrões alimentares e fatores demográficos e socioeconômicos de crianças de um a seis anos na cidade de Pelotas, Rio Grande do Sul, Brasil.

\section{Material e métodos}

Este trabalho utilizou o banco de dados do estudo Efeito da Fortificação de Farinhas com Ferro sobre Anemia em Pré-escolares 17, coletado em 2008 por meio de um estudo transversal, de base populacional, que investigou crianças de um a seis anos residentes na cidade de Pelotas. Este estudo utilizou a amostra que compôs a última das quatro avaliações de uma série temporal, que objetivou medir o efeito da fortificação das farinhas de trigo e milho com ferro e ácido fólico sobre a prevalência de anemia em pré-escolares. Aspectos metodológicos dos inquéritos realizados anteriormente estão publicados 17,18.

As mães ou responsáveis pelas crianças foram entrevistados no domicílio por nutricionistas previamente treinados. Todas as crianças de um a seis anos residentes no domicílio sorteado eram elegíveis para o estudo. Foram coletadas informações sobre as características demográficas das crianças (sexo, idade e cor da pele observada pelo entrevistador) e socioeconômicas da família (renda familiar atual em reais, escolaridade dos pais em anos completos de estudos e número de moradores no domicílio).

A amostra necessária para o trabalho original era de 900 crianças 17, no entanto, foram encontradas 850 nos domicílios sorteados e avaliadas 799. Neste trabalho, as crianças menores de um ano ( $n=132$ ) foram excluídas. Essa exclusão se fez necessária baseando-se no fato de que as crianças no primeiro ano de vida têm um consumo alimentar distinto das maiores 19. A amostra final englobou crianças com idades entre um e seis anos $(\mathrm{n}=667)$.

Para investigar o hábito alimentar foi aplicado à mãe ou ao responsável pela criança um questionário de frequência alimentar (QFA), referente ao consumo no último ano. O QFA foi construído conforme a metodologia proposta por Cardoso 20, contendo 56 itens, elaborado com base nos alimentos mais consumidos em recordatórios de $24 \mathrm{~h}$, aplicado às mães de crianças da mesma faixa etária, coletados na terceira avaliação do estudo original. O responsável pela criança tinha de responder quantas vezes os alimentos eram consumidos (0 a 10 vezes) por dia, semana, mês ou ano. Dessa forma, foi avaliada a frequência de consumo de alimentos mais incidentes pelas crianças. De posse dessa informação, efetuamos as transformações para frequências mensais da seguinte forma: o consumo diário foi multiplicado por 30,42; o consumo semanal foi multiplicado por quatro e o consumo anual foi dividido por 12. A escolha de trabalharmos com a frequência mensal também teve o intuito de minimizar a perda de infor- 
mação referente aos alimentos mais raramente consumidos.

Foi estipulada, arbitrariamente, uma frequência máxima aceitável para o consumo dos alimentos, com base nas porções dos grupos alimentares recomendadas pela pirâmide alimentar infantil (Departamento de Nutrologia, Sociedade Brasileira de Pediatria. Pirâmide Alimentar. http://www.sbp.com.br/pdfs/1429e1 cartaz_Piramide.pdf, acessado em Fev/2013). Consideramos aceitável a ingestão de alimentos de refeições principais, tais como feijão, arroz, carne, legumes e vegetais, até três vezes por dia; frutas, até cinco vezes por dia; e leite e achocolatados, até oito vezes por dia. Da amostra geral de crianças, apenas $1,2 \%$ ultrapassou essa frequência, sendo que para estas foi atribuído o valor máximo aceitável.

O consentimento por escrito da mãe ou responsável foi obtido antes da coleta das informações. Este estudo foi submetido e aprovado pelo Comitê de Ética da Faculdade de Medicina da Universidade Federal de Pelotas.

\section{Análise dos dados}

A fim de identificar os padrões alimentares, dos 56 itens que compuseram o QFA, dois foram excluídos por apresentarem uma frequência de consumo inferior a $10 \%$. Os alimentos restantes foram agrupados e classificados em 35 grupos (Tabela 1), de acordo com as suas características nutricionais e a correlação de Pearson entre os seus itens alimentares. O sorvete foi excluído nos dois grupos etários por não ter sido considerada a época de consumo (se consumido apenas no verão ou durante todas as épocas do ano) no momento da entrevista, o que poderia superestimar o consumo anual.

A análise fatorial para identificação dos padrões alimentares foi realizada por meio da PCA. O método de PCA é uma análise estatística multivariada, que utiliza informações relatadas nos instrumentos de avaliação do consumo alimentar para identificar dimensões subjacentes comuns (fatores ou padrões) do consumo de alimentos. Ela agrega alimentos ou grupos de alimentos em função do grau de correlação que os itens alimentares apresentam entre si no conjunto de dados. Para a avaliação da aplicabilidade do método foi estimado o coeficiente de Kaiser-Meyer-Olkin (KMO). O KMO verifica a existência e o peso das correlações parciais, não sendo aceitos valores abaixo de 0,6 21. Posteriormente, o teste de esfericidade de Bartlett foi aplicado para aferir a qualidade das correlações entre as variáveis. Um valor de p igual ou menor que 0,05 indica que o modelo fatorial é adequado para a análise dos dados 21. Depois foi realizada a análise fatorial exploratória, que gerou combinações lineares dos alimentos, possibilitando a simplificação dos dados. Em seguida, realizou-se a rotação ortogonal (Varimax) para examinar a estrutura (padrão) fatorial exploratória do QFA. Tal medida permite que os fatores resultantes não sejam correlacionados, levando a uma melhor interpretabilidade dos padrões 21 .

O número de fatores (padrões) que melhor representa os dados foi escolhido primariamente

\section{Tabela 1}

Quadro apresentando os alimentos/grupos de alimentos utilizados na análise de componentes principais.

\begin{tabular}{|c|c|}
\hline Grupo & Alimentos \\
\hline 1 & Cenoura cozida + crua \\
\hline 2 & Chuchu + brócolis + alface \\
\hline 3 & Abóbora \\
\hline 4 & Couve \\
\hline 5 & Beterraba cozida + beterraba crua \\
\hline 6 & Pera + mamão \\
\hline 7 & Carne com e sem osso + frango + fígado \\
\hline 8 & Ovo \\
\hline 9 & Feijão \\
\hline 10 & Pão \\
\hline 11 & Manteiga + margarina \\
\hline 12 & Arroz e massa \\
\hline 13 & Café solúvel \\
\hline 14 & Açúcar \\
\hline 15 & Suco artificial \\
\hline 16 & Salgadinho chips \\
\hline 17 & Presunto + mortadela + salsicha + linguiça \\
\hline 18 & Refrigerante \\
\hline 19 & Chocolate + brigadeiro + geleia \\
\hline 20 & Bala \\
\hline 21 & Maionese \\
\hline 22 & Bolo \\
\hline 23 & Leite integral + iogurte + queijo \\
\hline 24 & Achocolatado em pó \\
\hline 25 & Biscoito doce \\
\hline 26 & Suco natural \\
\hline 27 & Biscoito salgado \\
\hline 28 & Mandioca + amido de milho + farinha láctea \\
\hline 29 & Gelatina \\
\hline 30 & Batata \\
\hline 31 & Bergamota \\
\hline 32 & Laranja \\
\hline 33 & Banana \\
\hline 34 & Maçã \\
\hline 35 & Tomate \\
\hline
\end{tabular}


com base no teste gráfico de Cattel (scree plot ou gráfico de barreira), no qual os pontos de maior declive indicaram o número apropriado de componentes retidos 21 . A interpretabilidade das cargas fatoriais foi também considerada na escolha do número de componentes. Alimentos ou grupos de alimentos cujas cargas de saturação do fator ficaram acima de 0,4 foram avaliados como tendo uma forte associação com o componente, fornecendo melhor informação para a descrição de um padrão alimentar.

A consistência interna das dimensões do QFA foi avaliada, sendo considerado aceitável um índice alpha de Cronbach $\geq 0,6022$.

As análises estatísticas para a identificação dos padrões alimentares foram realizadas no programa SPSS versão 13.0 (SPSS Inc., Chicago, Estados Unidos). As análises univariadas, utilizando-se as variáveis sexo, cor da pele, escolaridade e renda familiar; e bivariadas cruzando as variáveis dos padrões alimentares identificados com sexo, escolaridade e renda familiar foram realizadas com o programa Stata versão 11.0 (Stata Corp., College Station, Estados Unidos). As análises bivariadas foram realizadas usando-se o teste de KruskalWallis com um nível de significância de 0,05 .

\section{Resultados}

As características demográficas e socioeconômicas das crianças estão apresentadas na Tabela 2. Foram estudadas 667 crianças, após a exclusão das 132 menores de um ano. A amostra se caracterizou principalmente por crianças do sexo masculino, com cor da pele branca, filhos de mulheres com nove ou mais anos de estudos e provenientes de famílias com renda mensal entre um e menos de três salários mínimos.

O coeficiente KMO calculado para a amostra de crianças foi de 0,72 , sugerindo que é adequado aplicar componentes principais aos dados.

A PCA identificou cinco padrões alimentares na amostra de crianças de um a seis anos. Os padrões identificados foram nomeados de acordo com o perfil dos alimentos que apresentaram as maiores cargas fatoriais.

Os padrões alimentares identificados foram "vegetais", "tradicional”, "guloseimas e embutidos", "lanches" e "frutas", que explicaram 34,3\% da variância total dos dados. O padrão "vegetais" foi composto principalmente pelo consumo de cenoura, chuchu, brócolis, alface, abóbora, couve e beterraba). As frutas pera e mamão, agrupadas a priori, apresentaram uma alta carga fatorial dentro desse padrão. O padrão "tradicional" é caracterizado pelo consumo de feijão, pão, manteiga/margarina, arroz, massa, açúcar e café. O padrão "guloseimas e embutidos" registra o consumo de salgadinhos, embutidos, chocolate, brigadeiro, geleia, refrigerantes, bala, maionese e bolo. O padrão "lanches" representa o consumo de laticínios, achocolatados, biscoitos doce e salgado e suco natural. O padrão "frutas" está relacionado ao consumo das frutas bergamota, laranja, banana e maçã.

As cargas fatoriais e o percentual da variância explicada por cada um dos padrões alimentares identificados estão apresentados na Tabela 3, em que também estão os valores do alpha de Cronbach, utilizados para avaliar a consistência interna do grupo de variáveis.

$\mathrm{Na}$ Tabela 4 apresentamos as médias dos padrões alimentares de acordo com as características demográficas e socioeconômicas da amostra.

Na amostra de crianças, a maior escolaridade materna esteve associada com a maior aderência aos padrões "vegetais" ( $\mathrm{p}=0,02)$ e "frutas" ( $\mathrm{p}<$ 0,001 ), enquanto que a menor escolaridade (menos de 4 anos de estudos) esteve ligada à maior aderência ao padrão "tradicional” ( $\mathrm{p}<0,001$ ). Quanto à renda, a adesão aos padrões "vegetais" ( $p<0,001$ ) foi maior para as crianças cuja renda familiar era de seis ou mais salários mínimos, e para os padrões "lanches" ( $\mathrm{p}=0,004)$ e "frutas" $(\mathrm{p}=0,000)$ a adesão foi maior entre as crianças com renda familiar de 3 a menos de 6 salários. $O$ padrão "tradicional" ( $p=0,000$ ) teve maior adesão entre as crianças cuja a renda familiar esteve de 1 a menos de 3 salários mínimos.

\section{Discussão}

A formação de hábitos alimentares saudáveis a partir da fase pré-escolar é fundamental, contribuindo para a manutenção de uma alimentação adequada nas fases subsequentes da vida 7 . Uma alimentação adequada evita carências nutricionais e contribui para a manutenção do peso corporal 23. A obesidade, quando adquirida durante a infância, tende a persistir na vida adulta, muitas vezes acompanhada de doenças crônicas relacionadas 6 .

Para essa faixa etária o presente estudo identificou cinco padrões alimentares. O primeiro padrão identificado foi o "vegetais", considerado um padrão alimentar saudável rico em vitaminas, minerais e fibras. A ingestão de alimentos desse grupo é de suma importância para crianças nessa faixa etária, pois além de contribuir positivamente para o crescimento e desenvolvimento, pode auxiliar na manutenção do peso corporal e na prevenção de doenças crônicas 23,24 . Outros 
Características demográficas e socioeconômicas das crianças de 1-6 anos. Pelotas, Rio Grande do Sul, Brasil, 2008.

\begin{tabular}{|c|c|c|}
\hline \multirow[t]{2}{*}{ Características } & \multicolumn{2}{|c|}{ Crianças de $1-6$ anos $(N=667)$} \\
\hline & $\mathrm{n}$ & $\%$ \\
\hline \multicolumn{3}{|l|}{$\operatorname{Sexo}(n=667)$} \\
\hline Masculino & 346 & 51,9 \\
\hline Feminino & 321 & 48,1 \\
\hline \multicolumn{3}{|l|}{$\operatorname{Cor}(n=664)$} \\
\hline Branca & 505 & 76,0 \\
\hline Não branca & 159 & 24,0 \\
\hline \multicolumn{3}{|c|}{ Escolaridade da mãe [anos] $(n=665)$} \\
\hline Até 4 & 101 & 15,2 \\
\hline $5-8$ & 261 & 39,2 \\
\hline 9 ou mais & 303 & 46,6 \\
\hline \multicolumn{3}{|c|}{ Escolaridade do pai ou padrasto [anos] $(\mathrm{n}=621)$} \\
\hline Até 4 & 98 & 15,8 \\
\hline $5-8$ & 277 & 44,6 \\
\hline 9 ou mais & 246 & 39,6 \\
\hline \multicolumn{3}{|c|}{ Renda familiar [salário mínimo] $(\mathrm{n}=665)$} \\
\hline Menos de 1 & 161 & 24,2 \\
\hline $1-2,99$ & 322 & 48,4 \\
\hline $3-5,99$ & 117 & 17,6 \\
\hline 6 ou mais & 65 & 9,8 \\
\hline \multicolumn{3}{|c|}{ Numero de moradores no domicílio $(n=667)$} \\
\hline $2-4$ & 488 & 73,2 \\
\hline $5-7$ & 148 & 22,2 \\
\hline 8 ou mais & 31 & 4,6 \\
\hline
\end{tabular}

Nota: o número máximo de observações desconhecidas foi 46 (6,9\%) para a variável Escolaridade do pai ou padrasto.

trabalhos, utilizando o mesmo tipo de análise e realizados com crianças entre quatro e dez anos, identificaram padrão alimentar similar, com o maior porcentual da variância entre os demais padrões alimentares identificados 25,26. Outros estudos mostraram padrões alimentares nomeados como "saudáveis" que continham, entre outros alimentos, itens alimentares com alta carga fatorial pertencentes ao grupo dos vegetais $9,13,27,28,29,30,31,32,33$.

O segundo padrão alimentar identificado foi o "tradicional", caracterizado por alimentos comumente consumidos na dieta do brasileiro. Esse é um padrão considerado monótono, pois embora integre itens alimentares importantes na dieta infantil como o arroz e o feijão, não inclui alimentos como vegetais e frutas, os quais são importantes fontes de micronutrientes e fibras. Padrões alimentares nomeados como tradicionais também foram identificados em vários estudos que utilizaram a PCA ou análise de clusters para avaliar a dieta de crianças, porém há dife- renças nos alimentos que compõem os padrões devido à cultura alimentar dos locais onde os estudos foram realizados 9,27,28,32,34,35.

O terceiro padrão identificado foi o "guloseimas e embutidos", composto por alimentos pouco nutritivos e de alta densidade energética, tais como salgadinhos, balas, chocolates, bolos e refrigerantes. Os alimentos que compõem esse padrão estão comumente relacionados ao aumento do peso corporal e do risco para o aparecimento de doenças crônicas como diabetes, hipertensão e doenças cardiovasculares. Além dos alimentos considerados guloseimas, esse padrão alimentar apresentou altas cargas fatoriais para os embutidos, como presunto, mortadela, salsicha e linguiça. Esses alimentos são calóricos e ricos em gordura saturada e sódio, cujo consumo está relacionado à incidência de dislipidemias e aterosclerose 36 . Vários estudos realizados com o propósito de identificar padrões alimentares em crianças têm identificado composição alimentar semelhante $25,26,28,29,32,34$. Um trabalho realizado 
Distribuição das cargas fatoriais dos alimentos/grupos de alimentos dos cinco padrões alimentares identificados para as crianças de um a seis anos. Pelotas, Rio Grande do Sul, Brasil, 2008.

\section{Alimentos/grupos de alimentos}

Crianças $>12$ meses

Padrões

Guloseimas e Lanches $\quad$ Frutas
embutidos

Cenoura cozida + crua

Vegetais

Tradicional

embutidos

Chuchu + brócolis + alface

0,718

0,678

0,642

0,546

Couve

Beterraba cozida + beterraba crua

0,465

0,458

0,288

Carne com e sem osso + frango + fígado

0,208

0,040

0,091

0,288

0,035

0,114

$-0,032$

$-0,236$

0,013

0,132

$-0,107$

0,021

$-0,066$

0,044

0,211

$-0,105$

$-0,281$

0,100

0,273

0,118

0,156

0,212

0,235

$-0,024$

0,077

0,068

0,211

0,186

8,0

8,0

0,69
0,065

0,137

0,028

0,013

0,021

$-0,147$

0,167

0,202

$-0,119$

0,185

0,110

0,102

0,037

0,133

0,207

0,613

0,517

0,491

0,472

0,465

0,443

0,431

0,220

0,262

0,117

$-0,055$

$-0,097$

$-0,294$

$-0,104$

$-0,024$

0,060

0,011

$-0,093$

0,159

0,041

0,075

7,4

15,4

0,61
0,137

$-0,002$

0,176

$-0,092$

0,125

0,279

0,041

$-0,068$

0,145

$-0,115$

$-0,132$

0,224

$-0,337$

0,338

$-0,001$

0,007

$-0,068$

0,171

0,060

$-0,005$

$-0,074$

0,010

0,719

0,520

0,426

0,418

0,408

0,381

0,312

0,293

0,023

$-0,068$

0,176

0,133

$-0,019$

6,4

28,2

0,51
$-0,001$

0,206

$-0,024$

0,125

0,178

0,042

0,134

0,067

$-0,034$

0,083

0,098

$-0,045$

0,006

0,030

0,154

$-0,027$

0,242

$-0,133$

0,008

0,006

0,109

0,016

0,198

0,214

$-0,070$

$-0,100$

0,226

0,044

0,046

$-0,169$

0,700

0,694

0,647

0,450

0,351

6,1

34,3

0,60

Nota: cargas fatoriais com valores maiores do que 0,40 estão em negrito. 
Escores médios dos padrões alimentares de acordo com as características demográficas e socioeconômicas das crianças de 1-6 anos. Pelotas, Rio Grande do Sul, Brasil, 2008. ( $N=667)$.

\begin{tabular}{|c|c|c|c|c|c|}
\hline \multirow[b]{2}{*}{ Características } & \multicolumn{5}{|c|}{ Padrões alimentares } \\
\hline & Vegetais & Tradicional & $\begin{array}{l}\text { Guloseimas } \\
\text { e embutidos }\end{array}$ & Lanches & Frutas \\
\hline Sexo & 0,12 & 0,82 & 0,31 & 0,40 & 0,62 \\
\hline Masculino & $-6,8$ & $-0,2$ & 5,6 & $-3,8$ & 2,7 \\
\hline Feminino & 7,3 & 0,3 & $-6,0$ & 4,1 & $-2,9$ \\
\hline Escolaridade da mãe (anos) & 0,00 & 0,00 & 0,08 & 0,19 & 0,00 \\
\hline Até 4 & $-32,2$ & 39,7 & $-13,6$ & 5,5 & $-4,36$ \\
\hline $5-8$ & 4,3 & 17,8 & 1,3 & $-10,4$ & $-13,3$ \\
\hline 9 ou mais & 6,8 & $-28,3$ & 3,2 & 7,2 & 26,7 \\
\hline Renda familiar (salário mínimo) & 0,00 & 0,00 & 0,52 & 0,00 & 0,00 \\
\hline Menos de 1 & $-19,9$ & 32,6 & 39,5 & $-23,1$ & $-22,0$ \\
\hline $1-2,99$ & $-2,11$ & 53,9 & $-0,8$ & 5,1 & 1,1 \\
\hline $3-5,99$ & 12,4 & $-19,7$ & $-6,0$ & 20,4 & 18,3 \\
\hline 6 ou mais & 39,9 & $-72,5$ & 4,3 & $-8,1$ & 14,5 \\
\hline
\end{tabular}

Nota: teste de Kruskal Wallis utilizando um nível de significância de 0,05. Valores dos escores foram divididos por 100 para facilitar a leitura da tabela.

por Gama et al. 14, no Rio de Janeiro, com famílias de baixo nível econômico, encontrou uma prevalência de 68,4\% de alterações no perfil lipídico e $10,7 \%$ de sobrepeso em crianças com idades entre cinco e nove anos. Esse mesmo estudo, utilizando PCA, identificou seis padrões alimentares, entre os quais dois que podem estar relacionados com os resultados de sobrepeso e dislipidemias encontrados, caracterizados pelo consumo de doces, biscoitos, bebidas industrializadas, frituras e embutidos.

O quarto padrão alimentar encontrado foi o "lanches". Esse padrão é caracterizado por alimentos normalmente consumidos entre as refeições principais, os quais nessa faixa de idade incluem leite, iogurte, achocolatados, biscoitos e suco natural. As crianças que aderem a esse padrão provavelmente não fazem as refeições principais adequadamente, substituindo-as por lanches rápidos. Essa é uma prática comum entre as crianças em idade pré-escolar, a qual pode contribuir para carências nutricionais e excesso de peso ${ }^{37}$. Muitos estudos mostraram padrão alimentar semelhante em crianças com idades entre três e onze anos, identificados por meio da PCA 28,29,30,38,39. A constituição alimentar desse padrão mostra-se mais saudável do que as apresentadas nos demais estudos revisados, os quais, entre outros alimentos, apresentam a inclusão de salgadinhos, doces, bebidas adocicadas e refrigerantes.
O quinto padrão alimentar encontrado foi o “frutas”. Assim como o padrão “vegetais”, identificado neste estudo, os benefícios da adesão ao padrão "frutas" estão relacionados ao seu teor de vitaminas, mineras e fibras. Trabalhos realizados com o propósito de identificar padrões alimentares, usando-se a PCA ou a análise de cluster, encontraram as frutas com altas cargas fatoriais, inseridas em padrões saudáveis ou em padrões caracterizados pelo consumo de frutas e vegetais especificamente 9,25,26,27,28,29,30,31,32,33.

Um resultado importante deste estudo é o fato de as carnes e ovos não terem atingido cargas fatoriais suficientes para saturar nos padrões alimentares selecionados para as crianças maiores de um ano. Isso sugere baixo consumo de proteínas de alto valor biológico, essenciais para um crescimento adequado 40 . A recomendação proteica durante a infância é maior do que para os adultos, havendo também a necessidade de maior proporção de aminoácidos essenciais. A ingestão de proteínas de alto valor biológico deve alcançar dois terços do total recomendado ${ }^{41}$. Outros estudos realizados com crianças identificaram as carnes contribuindo com altas cargas fatoriais nos padrões identificados 9,25,26,28,30,31,32,38. Um trabalho realizado por Farias Junior \& Osório 19, em Pernambuco, com crianças menores de cinco anos, destacou a carne entre os seis alimentos mais consumidos; no entanto, a carne foi consumida por apenas $36 \%$ das crianças, sendo o 
seu consumo positivamente associado à renda familiar. Segundo dados da PNDS, somente $30 \%$ das crianças entre 18 e 24 meses consomem carne diariamente 42 . $\mathrm{O}$ baixo consumo de carnes é extremamente preocupante, uma vez que no Brasil ainda se tem prevalências elevadas de anemia ferropriva 42 . Sendo a carne um dos alimentos fontes de ferro de alta biodisponibilidade na dieta infantil, o consumo diário deste alimento é essencial 43.

\section{Padrões alimentares e sua relação com as variáveis socioeconômicas e demográficas}

Os padrões alimentares "vegetais" e "frutas" foram positivamente associados à maior escolaridade materna e à renda familiar. Esses resultados sugerem que indicadores de melhores condições socioeconômicas contribuem positivamente na qualidade da alimentação de crianças menores de seis anos 44 . O padrão alimentar "tradicional" que se caracteriza por alimentos comumente presentes na dieta do brasileiro, teve uma forte associação com a menor escolaridade das mães e renda familiar. Um estudo realizado por Gatica 12, em Pelotas, com crianças que foram acompanhadas aos 12, 24 e 48 meses, também identificou o padrão alimentar "tradicional" positivamente associado a características indicativas de menor nível socioeconômico. Os padrões alimentares mais tradicionais da dieta brasileira são geralmente relacionados com a alimentação de famílias com baixo nível socioeconômico 12,15. Esses padrões geralmente não incluem alimentos considerados saudáveis, pertencentes aos grupos dos vegetais e das frutas. É evidente que a preocupação com o consumo de uma dieta mais adequada está nas famílias que têm maior renda e maior escolaridade materna 13,26,29.

É importante, no entanto, ressaltar que a adesão a um padrão alimentar não exclui, necessariamente, a adesão aos outros padrões. Neste tipo de análise os padrões alimentares podem não representar os ideais de dieta e, por serem específicos de uma amostra, podem não se repetir em outras populações 21 .
Uma das principais limitações do estudo na identificação de padrões alimentares está na subjetividade presente em diversas etapas da análise, tais como na escolha dos critérios utilizados para agrupar os alimentos, no número de fatores que serão retidos, no tipo de rotação a que os dados serão submetidos e na forma como os padrões identificados serão nomeados 45 . Como forma de minimizar os efeitos dessa limitação, neste estudo, as decisões tomadas durante todas as etapas da análise foram detalhadamente descritas. Outra limitação diz respeito ao método de avaliação do consumo alimentar por meio de QFA, que é dependente da memória. Por outro lado, a dieta nessa faixa etária tende a ser monótona e não sofrer grandes variações e, ainda segundo Willet 46 , as pessoas lembram melhor a dieta habitual do que a de um dia específico. Além disso, o instrumento possui várias vantagens, tais como simplicidade na análise, baixo custo, economia de tempo, e permitir a classificação dos indivíduos em categorias de consumo 47 .

\section{Conclusão}

O estudo identificou cinco padrões alimentares. Os “vegetais" e 'tradicional' foram os padrões que responderam com o maior percentual de variância nesta amostra de crianças. Maior escolaridade materna e renda familiar foram as características determinantes da adesão a padrões considerados saudáveis.

Os padrões alimentares identificados neste estudo mostraram, de forma geral, uma alimentação monótona na qual nem todos os grupos de alimentos estiveram presentes.

Um comportamento alimentar inadequado nessa fase da vida pode ser perpetuado, podendo ter influência direta no estado de saúde. Em virtude disso, a identificação e o conhecimento desses padrões alimentares em crianças em idade pré-escolar favorecem o subsídio de intervenções, no intuito de promover a educação nutricional ainda durante a infância. 


\section{Resumen}

El estudio tuvo como objetivo describir los patrones dietéticos para investigar su asociación con factores demográficos y socioeconómicos entre niños de uno a seis años en la ciudad de Pelotas. Se utilizó la correlación de Pearson para grupos de alimentos. Los patrones dietéticos se construyeron mediante el análisis de componentes principales (PCA). Las asociaciones se examinaron mediante pruebas Kruskal-Wallis $(\alpha=0,05)$. Asimismo, se evaluaron a 667 niños y se identificaron cinco patrones dietéticos: "hortalizas", "dulces y embutidos", "tradicional" (pan, mantequilla/margarina, arroz/pasta, café, azúcar), "aperitivos" (lácteos, chocolate, galletas y zumo) y "fruta". Los patrones "hortalizas" y "fruta" tuvieron mayor adhesión entre los niños cuyas madres tenían educación superior e ingresos altos. El patrón "tradicional" se observa más en niños cuyas madres tenían menos educación e ingresos medios. Los patrones "hortalizas" y "tradicional" presentaron mayor variación. Las condiciones socioeconómicas de las familias juegan un papel clave en la determinación de los patrones de la dieta de los niños.

Conducta Alimentaria; Niño; Preescolar; Análisis Factorial

\section{Colaboradores}

R. L. V. Souza e M. C. F. Assunção participaram do planejamento, análises estatísticas e revisão do artigo. S.W. Madruga contribuiu nas análises estatísticas e revisão do artigo. D. P. Gigante, I. S. Santos e A. J. D. Barros colaboraram na revisão do artigo.

\section{Agradecimentos}

Este estudo contou com o apoio financeiro do Fundo Nacional de Saúde, Ministério da Saúde - processo no 799/2003, e da CAPES, pela concessão de bolsa de estudos durante o período de realização do mestrado.

\section{Referências}

1. Monteiro CA, Conde WL. Tendência secular da desnutrição e da obesidade na infância na cidade de São Paulo (1974-1996). Rev Saúde Pública 2000; 34(6 Suppl):52-61.

2. Lacerda E, Cunha AJ. Anemia ferropriva e alimentação no segundo ano de vida no Rio de Janeiro, Brasil. Rev Panam Salud Pública 2001; 9:294-301.

3. Vasconcelos FAG. Tendências históricas dos estudos dietéticos no Brasil. Hist Ciênc Saúde-Manguinhos 2007; 14:197-219.

4. Batista Filho M, Assis AM, Kac G. Transição nutricional: conceitos e características. In: Kag G, Sichieri R, Gigante DP, organizadores. Epidemiologia nutricional. Rio de Janeiro: Editora Fiocruz/Editora Atheneu; 2007. p. 445-60.

5. Mikkila V, Rasanen L, Raitakari OT, Pietinen P, Viikari J. Consistent dietary patterns identified from childhood to adulthood: the cardiovascular risk in Young Finns Study. Br J Nutr 2005; 93:923-31.
6. Magarey AM, Daniels LA, Boulton TJ, Cockington RA. Predicting obesity in early adulthood from childhood and parental obesity. Int J Obes Relat Metab Disord 2003; 27:505-13.

7. Madruga SW, Araujo CL, Bertoldi AD, Neutzling MB. Tracking of dietary patterns from childhood to adolescence. Rev Saúde Pública 2012; 46:376-86.

8. Gaglianone CP, Taddei JAAC, Colugnati FAB, Magalhâes CG, Davanço GM, Macedo L, et al. Educação nutricional no ensino público fundamental em São Paulo, Brasil. Projeto reeducação aos riscos de adoecer e morrer na maturidade. Rev Nutr 2006; 19:309-20.

9. Northstone K, Emmett PM. Are dietary patterns stable throughout early and mid-childhood? A birth cohort study. Br J Nutr 2008; 100:1069-76.

10. Garcia RWD. A comida, a dieta, o gosto: mudanças na cultura alimentar urbana. [Tese de Doutorado]. São Paulo: Universidade de São Paulo; 1999. 
11. Newby PK, Tucker KL. Empirically derived eating patterns using factor or cluster analysis: a review. Nutr Rev 2004; 62:177-203.

12. Gatica G, Barros AJ, Madruga S, Matijasevich A, Santos IS. Food intake profiles of children aged 12, 24 and 48 months from the 2004 Pelotas (Brazil) birth cohort: an exploratory analysis using principal components. Int J Behav Nutr Phys Act 2012; 9:43.

13. D’Innocenzo S, Marchioni DML, Prado MS, Matos SMA, Pereira SRS, Barros AP, et al. Condições socioeconômicas e padrões alimentares de crianças de 4 a 11 anos: estudo SCAALA - Salvador/ Bahia. Rev Bras Saúde Matern Infant 2011; 11:41-9.

14. Gama SR, Carvalho MS, Chaves CRMM. Prevalência em crianças de fatores de risco para as doenças cardiovasculares. Cad Saúde Pública 2007; 23:2239-45.

15. Nobre, LN, Lamounier, JA, Franceschini, SCC. Padrão alimentar de pré-escolares e fatores associados. J Pediatr (Rio J.) 2012; 88:129-36.

16. Hu FB. Dietary pattern analysis: a new direction in nutritional epidemiology. Curr Opin Lipidol 2002; 13:3-9.

17. Assunção MC, Santos IS, Barros AJ, Gigante DP, Victora CG. Efeito da fortificação de farinhas com ferro sobre anemia em pré-escolares, Pelotas, RS. Rev Saúde Pública 2007; 41:539-48.

18. Assunção MC, Santos IS, Barros AJ, Gigante DP, Victora CG. Flour fortification with iron has no impact on anaemia in urban Brazilian children. Public Health Nutr 2012; 15:1796-801.

19. Farias Junior G, Osório M. Padrão alimentar de crianças menores de cinco anos. Rev Nutr 2005; 18:793-802.

20. Cardoso MA. Desenvolvimento, validação e aplicações de questionários de frequência alimentar em estudos epidemiológicos. In: Kag G, Sichieri R, Gigante DP, organizadores. Epidemiologia nutricional. Rio de Janeiro: Editora Fiocruz/Editora Atheneu; 2007. p. 201-12.

21. Olinto MT. Padrões alimentares: análise dos componentes principais. In: Kag G, Sichieri R, Gigante DP, organizadores. Epidemiologia nutricional. Rio de Janeiro: Editora Fiocruz/Editora Atheneu; 2007. p. 213-26.

22. Hair Jr. JF, Anderson RE, Tatham RL, Black WC. Análise multivariada de dados. 9a Ed. Porto Alegre: Editora Bookman; 2005.

23. Coordenação-Geral da Política de Alimentação e Nutrição, Secretaria de Atenção à Saúde, Ministério da Saúde. Guia alimentar para a população brasileira: promovendo a alimentação saudável. Brasília: Ministério da Saúde; 2005.

24. Miller P, Moore RH, Kral TV. Children's daily fruit and vegetable intake: associations with maternal intake and child weight status. J Nutr Educ Behav 2011; 43:396-400.

25. Wardle J, Sanderson S, Leigh Gibson E, Rapoport L. Factor-analytic structure of food preferences in four-year-old children in the UK. Appetite 2001; 37:217-23.

26. Moreira P, Santos S, Padrão P, Cordeiro T, Bessa M, Valente $\mathrm{H}$, et al. Food patterns according to sociodemographics, physical activity, sleeping and obesity in Portuguese children. Int J Environ Res Public Health 2010; 7:1121-38.
27. Pryer JA, Rogers S. Dietary patterns among a national sample of British children aged 1 1/2-4 1/2 years. Public Health Nutr 2009; 12:957-66.

28. North K, Emmett P. Multivariate analysis of diet among three-year-old children and associations with socio-demographic characteristics. The Avon Longitudinal Study of Pregnancy and Childhood (ALSPAC) Study Team. Eur J Clin Nutr 2000; 54: 73-80.

29. Craig LC, McNeill G, Macdiarmid JI, Masson LF, Holmes BA. Dietary patterns of school-age children in Scotland: association with socio-economic indicators, physical activity and obesity. Br J Nutr 2010; 103:319-34.

30. Aranceta J, Perez-Rodrigo C, Ribas L, Serra-Majem L. Sociodemographic and lifestyle determinants of food patterns in Spanish children and adolescents: the enKid study. Eur J Clin Nutr 2003; 57 Suppl $1: S 40-4$.

31. Shin KO, Oh SY, Park HS. Empirically derived major dietary patterns and their associations with overweight in Korean preschool children. Br J Nutr 2007; 98:416-21.

32. Northstone K, Emmett P. Multivariate analysis of diet in children at four and seven years of age and associations with socio-demographic characteristics. Eur J Clin Nutr 2005; 59:751-60.

33. Gubbels JS, Kremers SP, Goldbohm RA, Stafleu A, Thijs C. Energy balance-related behavioural patterns in 5-year-old children and the longitudinal association with weight status development in early childhood. Public Health Nutr 2012; 15:1402-10.

34. Feinstein L, Sabates R, Sorhaindo A, Rogers I, Herrick D, Northstone K, et al. Dietary patterns related to attainment in school: the importance of early eating patterns. J Epidemiol Community Health 2008; 62:734-9.

35. Ovaskainen ML, Nevalainen J, Uusitalo L, Tuokkola JJ, Arkkola T, Kronberg-Kippila C, et al. Some similarities in dietary clusters of pre-school children and their mothers. Br J Nutr 2009; 102:443-52.

36. Sposito AC. Caramelli B, Fonseca FAH, Bertolami MC, Afiune Neto A, Souza AD, et al. IV diretriz brasileira sobre dislipidemias e prevenção da aterosclerose: Departamento de Aterosclerose da Sociedade Brasileira de Cardiologia. Arq Bras Cardiol 2007; 88 Suppl 1: 2-19.

37. Berkowitz R. Obesity in childhood and adolescence. In: Walker W, Watkins JB, editors. Nutrition in pediatrics. 2nd Ed. London: Decker; 1997. p. 716-23.

38. Lioret S, Touvier M, Lafay L, Volatier JL, Maire B. Dietary and physical activity patterns in French children are related to overweight and socioeconomic status. J Nutr 2008; 138:101-7.

39. Ritchie LD, Spector P, Stevens MJ, Schmidt MM, Schreiber GB, Striegel-Moore RH, et al. Dietary patterns in adolescence are related to adiposity in young adulthood in black and white females. J Nutr 2007; 137:399-406.

40. Ctenas MLB. Crescendo com saúde II: guia de nutrição infantil. São Paulo: C2 Editora e Consultoria em Nutrição; 2003. 
41. Vitolo MR. Recomendações nutricionais para crianças. In: Vitolo MR, organizador. Nutrição da gestação ao envelhecimento. Rio de Janeiro: $\mathrm{Ru}-$ bio; 2008. p. 191-9.

42. Ministério da Saúde. Pesquisa nacional de demografia e saúde da criança e da mulher - PNDS 2006: dimensões do processo reprodutivo e da saúde da criança. Brasília: Ministério da Saúde; 2009.

43. Departamento de Atenção Básica, Secretaria de Atenção à Saúde, Ministério da Saúde. Dez passos para uma alimentação saudável: guia alimentar para crianças menores de dois anos: um guia para o profissional da saúde na atenção básica. 2a Ed. Brasília: Ministério da Saúde; 2010.

44. Robinson S, Marriott L, Poole J, Crozier S, Borland S, Lawrence W, et al. Dietary patterns in infancy: the importance of maternal and family influences on feeding practice. Br J Nutr 2007; 98:1029-37.
45. Martinez ME, Marshall JR, Sechrest L. Invited commentary: factor analysis and the search for objectivity. Am J Epidemiol 1998; 148:17-9.

46. Willet WC. Nutritional epidemiology. New York: Oxford University Press; 1998.

47. Pereira RA, Sichieri R. Métodos de avaliação do consumo alimentar. In: Kag G, Sichieri R, Gigante DP, organizadores. Epidemiologia nutricional. Rio de Janeiro: Editora Fiocruz/Editora Atheneu; 2007. p. 181-200.

Recebido em 24/Out/2012

Versão final reapresentada em 16/Mai/2013 Aprovado em 03/Jun/2013 